\title{
Anabases
}

ANABASES Traditions et réceptions de l'Antiquité

$24 \mid 2016$

Varia

\section{L'énigme de Delphes : Vassilis Alexakis lecteur de Plutarque}

Alain Ballabriga

\section{OpenEdition}

Journals

Édition électronique

URL : http://journals.openedition.org/anabases/5968

DOI : 10.4000/anabases.5968

ISSN : 2256-9421

Éditeur

E.R.A.S.M.E.

Édition imprimée

Date de publication : 10 novembre 2016

Pagination : 201-216

ISSN : 1774-4296

\section{Référence électronique}

Alain Ballabriga, "L'énigme de Delphes : Vassilis Alexakis lecteur de Plutarque », Anabases [En ligne], 24 |

2016, mis en ligne le 01 octobre 2019, consulté le 22 mars 2020. URL : http://

journals.openedition.org/anabases/5968; DOI : https://doi.org/10.4000/anabases.5968

(c) Anabases 


\section{L'énigme de Delphes : Vassilis Alexakis lecteur de Plutarque}

Alain BaLlabriga

$N$ é en 1943 à Athènes, Vassilis Alexakis s'installe à Paris en 1968 après le coup d'État des colonels en Grèce et se fait connaître comme dessinateur humoristique et chroniqueur pour le compte de plusieurs journaux et chaînes de radio. Il est aussi un romancier qui, après avoir d'abord écrit en français, décide de se remettre au grec en se replongeant dans les grands romanciers grecs qui ont marqué les années 60 du $\mathrm{XX}^{\mathrm{e}}$ siècle tels que Stratis Tsirkas et Kostas Tachtsis ${ }^{1}$. Il devient ainsi, à partir de 1980, un écrivain bilingue dont les romans paraissent désormais à la fois en grec et en français ${ }^{2}$.

C'est le cas en 1995 pour son roman La langue maternelle, couronné par le prix Médicis, ce qui marque une étape importante dans la reconnaissance de l'auteur. Dans ce roman, le narrateur, nommé Pavlos Nikolaïdis, apparaît comme un double de l'auteur : sans être écrivain, c'est un Grec vivant à Paris, auteur de dessins humoristiques et à cheval entre deux langues et deux cultures ${ }^{3}$. Son récit, à l'occasion d'un séjour en Grèce, déploie de multiples chaînes narratives entrelacées, qui sont reprises tout au long d'un roman d'une écriture simple mais dont la structure savante ne se découvre qu'à une deuxième lecture voire lors d'une étude de l'œuvre.

La chaîne narrative principale relate les étapes d'une enquête menée par le narrateur à propos d'une énigme de Delphes : que signifiait la lettre epsilon

1 Stratis Tsirkas (1911-1980) auteur de la trilogie romanesque Cités à la dérive (19601965) ; Kostas Tachtsis (1927-1988) auteur du Troisième anneau (1962).

2 Pour une bonne présentation d'ensemble de l'œuvre de Vassilis Alexakis, voir le récent ouvrage de Marianne Bessy (Bessy, 2011).

3 On peut lire dans Bessy, 2011, p. 131-138, une analyse de ce roman. 
figurant à l'entrée du temple d'Apollon à Delphes ? À l'occasion d'un entretien, l'auteur qualifie cette enquête d' “ un peu universitaire »5. L'auteur s'est en effet livré, on va le voir au cours de cette étude, a des recherches érudites, dignes d'un helléniste, sur l'epsilon de Delphes. Mais le narrateur étant présenté comme un profane en matière d'études grecques, le savoir acquis par l'auteur est dispensé au narrateur grâce à une série d'interlocuteurs compétents. Ces interventions savantes prennent place dans un contexte plus général qui permet à l'auteur/ narrateur de donner son sentiment sur les rapports de la Grèce moderne à la langue et à la culture de la Grèce ancienne. C'est pourquoi, avant d'en venir au détail de l'enquête, il m'a paru utile de traiter assez rapidement de deux questions connexes, à savoir le rapport du narrateur d'une part au grec ancien, d'autre part à la culture antique vue à travers une tragédie classique dont la représentation peut être confrontée à un spectacle de « revue ».

\section{Le narrateur et le grec ancien}

Avant de commencer ses recherches sur l'epsilon de Delphes, le narrateur se demande : “ Pourquoi ai-je commencé à m'intéresser à l'epsilon, moi qui n'ai jamais étudié sérieusement la langue ancienne? » (p. 50) ${ }^{6}$. Et plus bas, après une évocation, remplie de traits satiriques, d'une réunion littéraire et mondaine dans une librairie d'Athènes ${ }^{7}$, il énumère les noms de rue antiques du quartier Colonaki pour conclure : “ Colonaki me rappelle l'étendue de mon ignorance, me montre ses véritables dimensions, me tire par l'oreille comme le faisaient les plus sévères de mes enseignants qui étaient, en général, des professeurs de grec ancien » (p. 109).

Suivent plusieurs pages de réflexions sur les rapports du narrateur au grec ancien (p. 110-116). À l'école, les cours de grec ancien étaient mornes et ennuyeux. Par réaction, les élèves se défoulaient en ayant recours à la dérision. Circulait

$4 \quad$ Il est significatif que le titre de la traduction allemande de ce roman soit Das Rätsel von Delphi (L'Enigme de Delphes).

5 Entretien avec Marianne Bessy, à Athènes, les 5 et 6 janvier 2010, voir Bessy, 2011, p. 239.

6 Je donne la pagination de l'édition en français (Paris Fayard, 1995) à laquelle j'ajoute

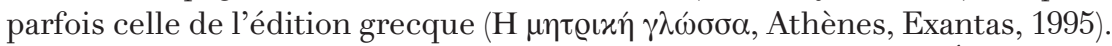

7 Il s'agit de la présentation à la presse d'un volume consacré à L'Éternelle Grecque où figurent la poétesse Sapho de Lesbos, l'historienne byzantine Anne Comnène (10831153), Laskarina Bouboulina (1771-1825) héroïne de la guerre d’indépendance de 1821, la romancière Pénélope Delta (1874-1941) qui se suicida lors de l'entrée des nazis à Athènes. Cette mise en perspective est caractéristique de la vision grecque de l'histoire nationale. 
ainsi en cachette une version pornographique de l'Iliade sur laquelle le narrateur revient plus loin (p. 210). Par exemple, à propos du cheval de bois, on pouvait lire :

“ Dans le creux de son ventre les Grecs s'étant mis

Par le trou de son cul observaient l'ennemi ».

Ce qui donne en grec :

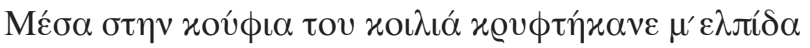

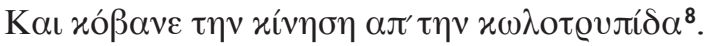

À la suite de cette évocation parodique, le narrateur confesse : “ L'Iliade et l'Odyssée ne nous intéressaient que sous forme de bandes dessinées ou de films. Je me souviens parfaitement de Kirk Douglas dans le rôle d'Ulysse. Je n'ai lu l'Odyssée que longtemps après avoir quitté l'école, alors que j'étais en train de perdre le souvenir de cette époque, dans une édition française de poche » (p. 110).

Mais, tout à son projet d'enquête sur l'epsilon de Delphes, le narrateur se décide à compléter ses lacunes en matière de littérature antique. Il achète une édition de l'Iliade en grec ancien avec sa traduction en grec moderne : « J'ai ouvert l'Iliade. Je n'avais pas vu le texte original depuis l'école. J'ai lu deux vers et je me suis aussitôt reporté à la traduction sur la page de gauche. J'ai lu encore un vers et sa traduction. J'ai continué ainsi pendant un moment : mon regard allait d'une page à l'autre comme si je suivais un match de tennis. J'ai dû reconnaître que l'original demeurait aussi hermétique pour moi qu'il l'était au lycée » (p. 113-114).

Cette tentative amène le narrateur à se souvenir de l'enseignement du grec ancien en Grèce dans les années 1950. En classe les élèves déchiffraient péniblement quelques passages d'auteurs anciens dont ils faisaient une analyse grammaticale. On ne lisait pas les œuvres en traduction, ce qui aurait été la seule manière d'en prendre une vue d'ensemble. En fait les jeunes Français qui, comme moi et certains de mes lecteurs, faisaient leurs humanités dans les années 1960, ont connu le même genre d'enseignement grammatical traditionnel. En Grèce s'y ajoutait l'illusion d'une continuité de la langue. Mais, même avec une pédagogie plus adaptée et efficace, l'étude du dialecte homérique reste une entreprise malaisée et de longue haleine, car il s'agit d'une langue poétique archaïque, très différente de la prose classique. La poésie homérique était déjà d'accès difficile pour les étudiants à l'époque de Plutarque par exemple, car ils étaient dans la situation de l'étudiant de nos jours face à des écrits vieux de plusieurs siècles.

$8 \quad$ P. 211 (français) et p. 184 (grec). On peut lire cette parodie de l'Iliade dans le recueil fait par Mary Koukoules du folklore érotique néo-grec (Koukoules, 1983, p. 22-28). Vassilis Alexakis traduit en alexandrins les vers grecs de quinze syllabes $(8+7)$. Cette forme de vers, appelé « vers politique », est caractéristique de la poésie grecque depuis l'épopée byzantine. 
On peut lire néanmoins des réflexions d'une autre portée plus loin dans le roman lorsque le narrateur est conduit à reprendre sa lecture de l'lliade : « J'ai tout de même trouvé ici ou là des termes que nous utilisons toujours dans le langage courant. J'ai éprouvé une sorte de respect pour ces mots qui, après avoir traversé tant de siècles, continuent à être disponibles et sont prêts à se lancer dans de nouvelles aventures » (p. 209). Certains de ces mots, qu'il énumère, sont aussi passés dans les langues européennes. Ces réflexions sérieuses se terminent par une pointe satirique comme souvent dans ce roman : “Apatè, l'escroquerie, qui réapparait sans cesse à la une des journaux, se trouve déjà dans l'Iliade » (p. 210). Effectivement le lecteur grec se trouve, face au grec ancien, dans une situation tout à fait spécifique faite d'un curieux mélange de familiarité et d'incompréhension, situation que peut apprécier l'helléniste non-hellène connaissant aussi le néogrec. C'est un peu le sentiment que peut procurer La chanson de Roland à un lecteur francophone mais peu versé en ancien français.

\section{Récit de deux spectacles : une tragédie classique et une revue satirique}

À l'occasion d'une manifestation culturelle, le narrateur, faisant entrer en scène une ministre de la culture, ex-comédienne, nommée Amalia Stathopoulou transposition romanesque de Melina Mercouri, dont un des prénoms était aussi Amalia - rappelle son interprétation d'Électre au théâtre d'Épidaure dans les années 1950. Il s'agit de l'Électre de Sophocle et de la scène où Électre, reconnaissant enfin son frère Oreste, s'exclame en grec moderne : “ $\Phi \omega v o u ́ \lambda \alpha \mu$ p que la version française rend par : “Voix tant attendue, tu es donc arrivée ? » La comédienne, future ministre, prononça cette réplique de telle façon que tout le monde fut bouleversé, y compris Oreste, qui en oublia du coup son texte ${ }^{9}$ !

Plus loin dans le roman, le narrateur lit dans un journal l'annonce d'une prochaine représentation de l'Électre de Sophocle. Un titre de la une reprend en majuscules la célèbre réplique avant d'ajouter : “ On entendra à nouveau la voix d'Amalia à Épidaure » (p. 166). Vers la fin du roman, en une dizaine de pages, le narrateur fait le récit de cette représentation. Mais auparavant il exprime son sentiment général sur les représentations de tragédies antiques : « Il y a une certaine servilité dans notre attitude face aux œuvres du passé, qui leur fait du tort, qui les trahit. Je pense aux troupes de théâtre, petites et grandes, qui jouent tous les étés, avec l'aide du ministère de la Culture, les mêmes tragédies, de manière souvent affligeante... » (p. 205).

Et quand il en vient à la description d'un de ces événements touristiques et mondains, son attitude est loin d'être recueillie et respectueuse (p. 380-391).

9 P. 95 (français) et p. 83 (grec). 
Ainsi il n'est pas concentré sur la représentation : il attend seulement la célèbre réplique (p. 386). Un ami lui raconte une histoire grivoise durant la représentation, à l'indignation des voisins (p. 388). Un peu plus sérieusement il se demande si le traducteur de la pièce en grec moderne n'a pas pris quelques libertés avec le texte de Sophocle (p. 390). Or on lit dans l'original au vers 1225 : ' $\Omega \phi \theta \dot{\varepsilon} \gamma \mu^{\prime}$, àфíxov; L'helléniste Paul Mazon, dans l'édition Budé, traduit : “ $O$ voix aimée, tu es venue à moi ! ». Le poète Jean Grosjean, dans la Pléiade, fait mieux : “ $\mathrm{O}$ voix enfin venue! »Quant à la traduction néo-grecque citée plus haut, elle est à mon sens assez réussie : $\phi \omega v o u ́ \lambda \alpha$ diminutif de $\phi \omega v \eta ́$ “ voix » rend bien l'émotion et l'amour fraternel d'Électre.

Mais après avoir prononcé la réplique tant attendue, l'actrice perd l'équilibre sur scène. Le narrateur a alors deux formules cruelles : «Elle a été trahie par ses jambes » et «Le temps lui a retiré sa faveur » (p. 390-391). Auparavant le narrateur

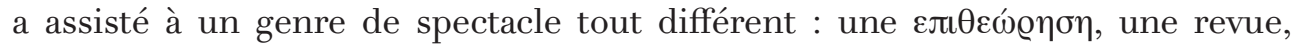
genre théâtral comprenant des monologues satiriques et des numéros de danse (p. 218-224). Dans cette revue par exemple, un des acteurs de la troupe revêt une paire de faux seins gigantesques, un tailleur noir et une perruque brune pour faire une parodie de la mère du premier ministre. Un autre acteur interprète le rôle d’un policier chargé des écoutes téléphoniques, écoutes qui permettent de mettre en lumière la corruption de certains hommes politiques.

Après la danse finale de la revue, le chef de la troupe se livre à une intervention sur la situation politique (milieu des années 1990) dans laquelle il déclare entre autres : “Ces aigrefins (les Européens) entendent nous donner des cours d'économie politique ! L'expansionnisme de la Serbie les préoccupe, mais pas celui de la Turquie ! Ils ne se soucient guère de Chypre, nos bons amis ! Ni des Kurdes d'ailleurs ! (p. 223). À la fin de ce discours, l'acteur, s'enroulant dans le drapeau national, s’écrie : “Vive la Grèce! » Mais les applaudissements sont moins nourris que pour les sketches précédents et les remarques du narrateur font bien voir qu'il s'agit d'un numéro d'acteur dont personne n'est dupe.

Un lecteur d'Aristophane fera bien sûr le rapport avec la " parabase ", cette partie de la comédie ancienne dans laquelle le chef de chœur s'adressait au public. Plus généralement il peut aussi sentir combien l'esprit de la revue est proche du théâtre aristophanesque. Or, justement, le genre de la revue a fortement influencé dans la Grèce moderne la réception du théâtre d'Aristophane, sous forme d'adaptations beaucoup plus populaires que les représentations de tragédies classiques. À ce propos, il est intéressant de savoir que le père de l'auteur est passé comme acteur par le Théâtre d'Art du grand metteur en scène Karolos Koun, célèbre en particulier pour des représentations d'Aristophane qui firent date ${ }^{10}$.

10 On peut lire sur cette question de la réception d'Aristophane dans la Grèce moderne l'excellent ouvrage de Gonda van Steen (VAN SteEn, 2000). 


\section{La quête sur l'E de Delphes}

Au début du séjour en Grèce raconté dans le roman, le narrateur se souvient avoir jadis découvert le mystère de l'E de Delphes en feuilletant un guide touristique chez son amie d'alors, pour passer le temps tandis qu'un plombier essayait vainement de réparer le lave-vaisselle ! Il avait alors appris dans ce guide qu'à l'entrée du temple d'Apollon à Delphes, outre les célèbres maximes des Sept Sages (“ Connais-toi toi-même », « Rien de trop »), figurait une grande lettre isolée, un epsilon, dont la signification reste inconnue malgré les nombreuses hypothèses formulées à son sujet (p. 31-33).

Comme je l'ai indiqué plus haut, le narrateur avoue ne s'être jamais intéressé à la Grèce ancienne et garde de mauvais souvenirs des cours de grec ancien au lycée. Mais il va trouver lors de son enquête des savants qui vont orienter sa recherche. Le premier d'entre eux, nommé Caradzoglou, est professeur à l'université d'Athènes. Il apprend au narrateur que la seule source antique sur cette énigme est constituée par un traité de Plutarque au début du II siècle ap. J.-C. Or Plutarque, bien que prêtre au sanctuaire de Delphes, ne livre que des hypothèses vaines (p. 105-106). Muni de cette information, le narrateur se rend dans une librairie pour acheter le traité de Plutarque. Il ne l'y trouve pas et finit par obtenir cet ouvrage dans la bibliothèque de l'École française d'Athènes. D'après la description de ce livre il doit s'agir de l'édition Budé procurée en 1974 par Robert Flacelière : on peut voir sur la première page deux photographies en noir et blanc de deux monnaies delphiques d'époque romaine sur lesquelles figure le $\mathrm{E}$ entre les colonnes du temple (p. 128).

Mais le narrateur ne se plonge pas d'emblée dans la lecture de ce traité. Il consulte toute une série de documents dont il tire des informations et des remarques originales, par exemple à propos de la photographie représentant la découverte en 1894, un siècle avant l'écriture du roman, de la statue d'Antinoos, le favori de l'empereur Hadrien (p. 131-134). Ce n'est qu'après une vingtaine de pages de réflexions diverses - ces sortes de digressions contribuent d'ailleurs à l'intérêt du roman - que le narrateur donne du traité de Plutarque une analyse qui prend en compte l'apparition du christianisme au temps de Plutarque : «Plutarque, qui est mort en 126, n'a jamais entendu parler du Christ. Il ignore le dieu nouveau qui prend son essor, mais il est bien obligé de constater qu'Apollon est à son déclin... Son texte, qui a la forme d'un dialogue platonicien, est d'abord un éloge d'Apollon. Les interprétations de l'epsilon qu'il propose plaident en faveur d'un dieu qui, à coup sûr, ne mérite pas de disparaitre. Il écrit pour sauver son dieu. Il se penche sur le si interrogatif afin d'exalter la capacité d'Apollon à résoudre les énigmes puis sur le si utilisé dans la dialectique, pour rappeler que le seigneur de Delphes est le fondateur de cet art. » (p. 158-159)" ${ }^{11}$. Quel rapport, peut se demander le lecteur,

1 Sur Delphes et le christianisme on trouve des réflexions semblables dans un texte de 
entre la conjonction « si » (en grec ancien ci ) et l'epsilon? Il y a là un vrai problème qui va s'éclairer sous peu.

Auparavant le narrateur a fait observer que les questions posées à l'oracle étaient d'ordinaire simples : “ Elles étaient posées par des particuliers qui cherchaient à savoir s'ils devaient se marier, prendre le bateau, s'expatrier... cultiver la terre. Selon Plutarque, l'epsilon pourrait désigner la conjonction ei, si, qui introduisait toutes ces questions. »(p.142-143) La version grecque du roman

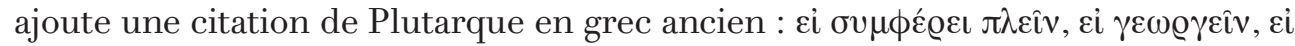

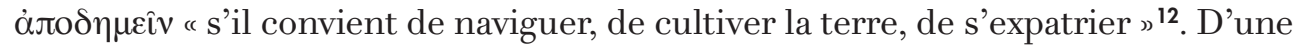
façon générale les citations en grec ancien sont moins exotiques dans un texte en grec moderne que dans une autre langue. On a affaire, j'y reviendrai plus bas, à deux états d'une même langue dans un même alphabet. En outre, dans le cas présent, la citation est compréhensible pour un lecteur grec peu versé en grec ancien, ce qui n'est pas le cas de la réplique d'Électre plus haut citée.

Par ailleurs, à la différence d'un helléniste, le narrateur entretient avec Plutarque une relation pour ainsi dire personnelle et familière, somme toute assez proche d'un Montaigne par exemple. Ainsi, à propos de l'expatriation, il dit un peu plus loin : ‘ Je l'ai retenue (cette question sur l'expatriation) car elle m'a beaucoup préoccupé, un été, il y a vingt-quatre ans. Elle me tourmentera peut-être encore, un jour. Je ne sais pas si je retournerai en France. Il se peut que ma vie là-bas se soit achevée ... » (p. 164). Et un peu plus loin encore : “ On quittait donc la Grèce déjà au temps de la Pythie. Mon départ pour l'étranger n’a été que la répétition d'une très vieille scène. » (p. 214). Mais pour en revenir à la forme ei, qui est en fait d'abord le nom de l'epsilon comme on va le voir sous peu, elle peut encore s'interpréter, entre autres, comme une forme du verbe “ être » : “L'epsilon serait l'initiale du verbe être, de la deuxième personne du présent plus précisément, et signifierait « tu es » (cela se dit encore ei)... La formule « tu es » incite à réfléchir sur la nature divine, à comprendre que seul Apollon existe réellement ... L'epsilon serait un traité de théologie tenant en une seule lettre. »(p. 159-160).

Un peu plus loin, le narrateur se pose une question en fait capitale et dont l'examen est susceptible de dissiper enfin l'embarras du lecteur sur le rapport de la graphie ei et de l'epsilon : “Si l'epsilon était l'initiale d'un mot aussi court que ei, pourquoi ne s'est-on pas donné la peine de l'écrire en entier? » (p. 162). En fait, le narrateur (mais aussi l'auteur) a été victime de l'édition consultée à la bibliothèque de l'École française d'Athènes. Il faut savoir en effet que Robert Flacelière avait pris le parti fort discutable de corriger systématiquement la tradition manuscrite

Georges Séféris écrit en 1961 (SEFERIs, 1987, p. 58 et 60-61). On peut noter d'autres ressemblances qui résultent peut-être de ce que Vassilis Alexakis connaissait ce texte. 
du traité de Plutarque en écrivant un seul epsilon là où on lit régulièrement ei. À commencer par le titre qui est, en majuscules, ПEPI TOY EI TOY EN $\Delta$ E $\Lambda$ ФI $\Sigma$ et non ПEPI TOY E TOY EN $\triangle \mathrm{E} \Lambda \Phi O I \Sigma$ comme dans l'édition Budé.

Or on peut trouver une explication de cette graphie dans un autre ouvrage consulté à la bibliothèque de l'École française. Il s'agit du Trésor de la langue grecque publié en 1572 par Henri Estienne (1531-1598) et réédité par l'imprimeur Firmin Didot (1764-1836) au début du XIX ${ }^{\mathrm{e}}$ siècle. Voici ce que nous dit le narrateur : « Je l'ai ouvert à la lettre E. Celle-ci est encadrée par une gravure qui représente un homme sur le point de rendre l'âme. Il est dans son lit, la tête rejetée en arrière. Une religieuse s'occupe de lui, alors que trois hommes l'observent, debout au pied de son lit. Pourquoi l'epsilon est-il accompagné d'un si triste tableau? Dois-je supposer qu'il est inspiré par le mot étimothanatos, moribond? » (p. 253) Plus loin le narrateur se demande : “ Le moribond qu'on voit sur la gravure qui illustre la lettre E dans le Trésor de la langue grecque serait-il son auteur, Henri Estienne? » (p. 289). Effectivement, comme l'indique une légende en latin, la gravure représente Henri Estienne sur son lit de mort dans un hôpital de Lyon en mars 1598 .

Si l'on consulte à son tour le Trésor d'Estienne, on peut s'aviser que l'article consacré à la lettre E informe en fait le lecteur que la graphie $\varepsilon$ ı était le nom de la

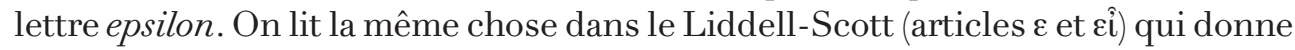
deux références fondamentales : un passage du Cratyle de Platon (437a7) où l'on trouve la graphie ei pour epsilon et un autre du grammairien Hérodien $\left(\mathrm{II}^{\mathrm{e}} \mathrm{s}\right.$. ap. J.-C.) selon lequel « la prononciation du ı est le nom de la lettre $\varepsilon{ }^{13}$.

Il faut savoir en effet qu'en la matière la prononciation “ érasmienne » du grec ancien, soit eï pour la graphie $e i$, est particulièrement trompeuse. Déjà à l'époque de Platon ( $\mathrm{IV}^{\mathrm{e}} \mathrm{s}$. av. J.-C.) la graphie ei notait un é long fermé tandis qu'à l'époque romaine le $e i$ se prononçait $i$ comme en grec moderne. Voilà pourquoi le nom $e i$ de l'epsilon se prononçait é au temps de Platon et i au temps de Plutarque. C'est à première vue un peu bizarre mais cela résulte simplement de l'évolution de la prononciation du grec au cours des temps hellénistiques et il n'y a aucune raison de corriger sur ce point le texte de Plutarque ${ }^{14}$. Quant au nom epsilon, qui nous est familier, il est plus récent que la forme ei (donc d'abord prononcé é puis i). Cette dénomination apparaît à époque romaine pour distinguer deux sortes de é : celui qui s'écrivait $\alpha \iota$ et celui qui s'écrivait $\varepsilon$. A l'époque de Plutarque la diphtongue $\alpha \iota$,

13 Herodiani Technici Reliquiae, ( ed. A. Lenz, Leipzig, 1867-1870), vol. 2, p. 390 : тฑेv

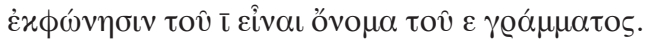

14 C'est ainsi à juste titre que l'éditeur du traité dans la Loeb Classical Library (1 ${ }^{\text {ére éd. }}$ 1936, vol. V, p. 198-199) garde le titre ПEPI TOY EI TOY EN $\Delta \mathrm{E} \Lambda \Phi O I \Sigma$ tandis que le titre anglais est THE E AT DELPHI. Quant à Jacques Amyot (1513-1593) dans sa traduction des Euvres morales (1572), il intitulait le traité Sur l'EI de Delphes. 
qui se prononçait ä̈ en grec classique, se prononçait é comme en grec moderne. Le $\alpha$ e était appelé “ é diphtongue » et le $\varepsilon$ “ epsilon » c'est-à-dire “ é simple ». De même l'upsilon doit son nom au fait que l'ancienne diphtongue se prononçant ü il fallait distinguer un « ü diphtongue » et un « ü simple ».

Or notre roman évoque le problème de la prononciation du grec ancien à l'occasion d'une conversation entre le narrateur et le bibliothécaire de l'École française, nommé François Bouchard, dans une taverne du quartier d'Exarchia. Ce personnage, deuxième savant à guider le narrateur profane, est un helléniste philhellène : il aime la vie à la grecque et parle parfaitement le grec moderne (p. 164-168). Mais il tente de persuader le narrateur « qu'il est exclu que l'epsilon de Delphes se soit substitué par erreur à la diphtongue ai, car les anciens prononçaient séparément les deux lettres, ils lisaient aïnigma» (p. 167). Quelques lignes plus bas, il fait valoir que : “ Le bêlement est transcrit en grec ancien par la syllabe $\beta \hat{\eta}, b \hat{e}$, que vous lisez vi aujourd'hui ». Et le narrateur, docile, de confesser aussitôt : “ Je ne savais ni que le bêta se prononçait $b$ et non $v$, ni que l'êta se lisait $\hat{e}$. Mes professeurs rejetaient avec mépris le point de vue d'Érasme sur la prononciation du grec ancien qui allait à l'encontre de leur propre théorie sur la non-évolution de la langue » (p. 167-168).

Que penser de ces propos? À la différence du narrateur, peu versé en grec ancien, l'auteur pourrait ne pas prendre les affirmations de l'helléniste français pour argent comptant. Dans un essai publié seize ans avant notre roman, il donnait en effet une longue citation du linguiste grec Georges Babiniotis dont voici les dernières lignes : “ Le mot $\beta \hat{\eta} \mu \alpha$ (tribune) que nous prononçons aujourd'hui vima, les anciens le prononçaient bèma. Ces changements déconcertent les hellénistes étrangers qui lisent le grec ancien avec la prononciation érasmienne. Cette prononciation, valable pour une certaine période de l'histoire de la langue, ne l'est pas pour toutes. On ne prononçait pas le grec de la même façon à l'époque d'Homère, de Platon et de Polybe. C'est une erreur de lire Platon avec la prononciation actuelle, mais c'est aussi une erreur de lire l'Évangile avec la prononciation érasmienne. Dans les premiers siècles de notre ère on prononçait de la même façon qu'aujourd'hui. D'où l'idée, qui a trouvé un écho favorable à l'étranger, de substituer à la prononciation érasmienne la prononciation actuelle, puisque ni l'une ni l'autre ne sont valables pour toutes les époques. Ce changement présenterait l'avantage de rendre accessible aux hellénistes étrangers le grec moderne, de rapprocher en somme les hellénistes et les Hellènes „ ${ }^{15}$.

Personnellement je suis entièrement d'accord avec Georges Babiniotis. Quant à l'auteur, Vassilis Alexakis, je serais enclin à croire que, dans les propos qu'il prête à la fois au narrateur et au bibliothécaire de l'École française, il faut voir une discrète et gentille ironie : il met un propos qu'il sait contestable dans la bouche d'un 
personnage qu'il présente comme un sympathique philhellène. Dans la version grecque du roman cette ironie se marque par un détail lexical relatif au bê bê des moutons qui, soit dit en passant, se trouve attesté dans deux fragments comiques de Cratinos et d'Aristophane comme en font foi les dictionnaires Bailly et LiddellScott. Alors que la version française parle de " bêlement ", la version grecque (p. 147) parle du $\beta \varepsilon ́ \lambda \alpha \sigma \mu \alpha$ des moutons, ce qui se prononce velazma. Autrement dit en français les moutons « bêlent » mais en grec ils « vêlent » (velazoune). On ne peut donc considérer le bê bê des moutons comme une onomatopée intemporelle et universelle !16

\section{Lire Plutarque}

En plus de l'édition bilingue (grec ancien/français) du traité de Plutarque procurée par Robert Flacelière, le narrateur, Pavlos Nikolaïdis, consulte une édition grecque bilingue (grec ancien/grec moderne) : « J'ai lu Plutarque en grec ancien et en grec moderne. Je le comprends très peu, si je ne lisais que le texte original. Plusieurs mots me sont familiers, mais il y a peu de phrases composées uniquement de mots que je comprends... Je suis incapable, naturellement, de porter un jugement sur le style de l'auteur. La traduction en grec moderne est plutôt ennuyeuse. Plutarque m'a rappelé les voix oubliées de mes professeurs » (p. 161-162). Or ce propos contraste fortement avec ce que l'auteur avait écrit dans l'essai ci-dessus mentionné : “ Un grec d'aujourd'hui, même s'il ne connaît que la langue parlée, peut lire sans trop de difficulté un texte de Xénophon. Un étranger ayant fait des études de grec trouvera dans les journaux actuels bien des mots familiers. Au fil des siècles, la langue et sa prononciation ont, bien sûr, évolué. Il n'en reste pas moins que le grec moderne n'est pas une langue nouvelle issue du grec ancien comme l'italien l'est du latin. Il s'agit bien de la même langue. C'est dans la langue de Platon que les Grecs achètent leur voiture, prennent leur billet d'avion et négocient leur adhésion à la C.E.E. »17.

À mon sens les sentiments éprouvés à la lecture de Plutarque sont plus proches de la vérité que les affirmations hasardeuses de l'essai. Certes Xénophon est un auteur plus facile que Plutarque ${ }^{18}$. Certes encore le grec moderne est plus proche

16 Sur cette question, je me permets de renvoyer à Ballabriga, 2006, p. 66.

17 Alexakis, 1979, p. 74.

18 Il était aussi un auteur facile pour les lecteurs d'époque romaine. On possède à cet égard un document remarquable de Dion de Pruse (30-116), contemporain de Plutarque

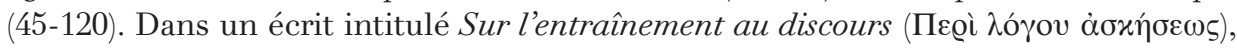
il donne des conseils de lecture à un personnage sans doute de quelque importance mais qui n'a pas eu la chance de faire de solides études dans sa jeunesse. Après avoir énuméré les grands classiques de la poésie et de la prose, d'Homère à Démosthène, 
du grec ancien que l'italien ne l'est du latin. Néanmoins le grec ancien n'est pas pour autant plus facile pour un grec que le latin ne l'est pour un italien. En fait les évolutions qui ont conduit du grec ancien au grec moderne sont analogues à celles qui ont conduit du latin à l'italien. C'est au cours du processus d'apprentissage d'une langue ancienne que la langue maternelle de l'étudiant peut colorer différemment le sentiment de familiarité. Un latiniste italien perçoit le latin autrement qu'un latiniste français dont la langue est plus éloignée du latin. C'est ce qui explique que beaucoup de latinistes italiens continuent de prononcer le latin à l'italienne et non à la restituée comme les Français, ce qui les rapproche des hellénistes grecs. En France, la situation est différente de celle de l'Italie et de la Grèce. Les sentiments du latiniste italien et de l'helléniste grec se rapprocheraient plutôt de ceux du médiéviste français : pour lui l'ancien français est senti un peu comme le grec ancien pour un helléniste grec. Le lecteur ordinaire, qui n'a pas étudié l'ancien français, doit avoir recours à des traductions en français moderne.

Quant aux éditions bilingues en grec ancien/moderne elles sont aussi comparables aux éditions bilingues en ancien français/français moderne. La traduction est faite dans un état plus récent de la langue et non dans une langue tout à fait étrangère. Mais l'écart entre la version originale et la traduction peut être plus ou moins grand selon la nature du texte à traduire. Le grec de Plutarque étant notoirement difficile, la traduction en grec moderne sera plus éloignée de la lettre du texte original. Par contre une traduction du Nouveau Testament, à peu près contemporain de Plutarque, pourra coller d'avantage à l'original, qui est dans un grec plus simple et plus proche du grec moderne. Et le lecteur d'une édition bilingue (grec ancien/moderne) du Nouveau Testament aura le sentiment d'une remarquable proximité entre le grec ancien et le grec moderne. Par ailleurs la traduction du grec ancien vers une langue moderne est une opération tout à fait spécifique, très différente de la traduction du grec moderne vers une autre langue. L'opération est d'abord plus difficile du fait de l'éloignement de la langue ancienne. Mais le traducteur actuel n'est pas dans la situation d'un étudiant qui fait une version grecque. Dans la plupart des cas, le traducteur du XX ${ }^{\mathrm{e}}$ siècle est en mesure d'étudier les traductions antérieures. Pour l'auteur de Sur l'EI de Delphes on peut remonter jusqu'à celle de Jacques Amyot, qui permit à Montaigne de lire Plutarque “ depuis qu'il est françois » ${ }^{19}$. La compréhension de Plutarque est ainsi le résultat d'un long processus historique depuis la Renaissance.

il termine par les Socratiques en insistant essentiellement sur Xénophon car Platon est trop difficile (\$14-18). On retire de cet écrit l'impression qu'il suffirait de connaître à fond l'œuvre de Xénophon, en particulier l'Anabase, pour faire bonne figure dans l'élite de l'empire romain, en dehors du cercle des virtuoses passés à la postérité.

19 Montaigne, Essais, livre II, chap. X, Des livres. La traduction par Amyot des Euvres morales fut publiée en 1572, année qui correspond au début de la rédaction des Essais. 
Mais cela est généralement vrai des classiques grecs, dont la plupart sont d'accès difficile ${ }^{20}$. Avec une franchise assez rare dans la profession, un helléniste connu pour sa traduction d'Aristophane a pu ainsi écrire : “ Pour nous autres, une comédie d'Aristophane, c'est un texte et un texte difficile, devant lequel nous nous sentons terriblement seuls, dont nous ne pouvons prendre connaissance qu'avec un dictionnaire sous le coude et plusieurs commentaires sous les yeux, ou bien à travers l'écran d'une traduction nécessairement misérable „21 . Le même auteur faisait aussi justement remarquer : “Que reste-t-il pour nos oreilles de la façon dont 'chantait' la langue dans sa prononciation et son accentuation ? », en esquissant une comparaison avec la lyrique médiévale française ${ }^{22}$. Effectivement notre prononciation actuelle du français est très différente de la prononciation médiévale. À l'exception de quelques médiévistes musicologues, on ne peut guère faire sentir comme sonnait le français des XII $-\mathrm{XIII}{ }^{\mathrm{e}}$ siècles ${ }^{23}$ et l'on est dans la même situation face à la littérature française médiévale que les lecteurs d'époque romaine face à la littérature grecque classique, situation qui est d'ailleurs toujours et nécessairement celle des hellénistes ${ }^{24}$.

\section{L'« omphalos » de Papaloukas}

L'enquête sur l'epsilon de Delphes en compagnie de Plutarque prend fin au chapitre 4. Mais dans la suite du roman, aux chapitres 6 et 8 , les recherches du narrateur offrent au lecteur une sorte de rebondissement dans le cadre de l'archéologie delphique contemporaine. Reprenant ses lectures à la bibliothèque de l'École française, le narrateur fait une découverte qu'il vaut la peine de citer assez longuement :

“ J'ai pris au hasard un des volumes à reliure bordeaux, qui rendent compte des découvertes faites pendant les fouilles de Delphes. Il datait de 1927. Il me réservait une surprise de taille. Je savais qu'aucun des deux omphalos trouvés à

20 On a vite fait le tour des exceptions à recommander à un débutant, par exemple les romans grecs d'époque romaine et surtout l'admirable Lucien de Samosate.

21 Debidour, 1962, p. 22-23 ; voir aussi dans le même sens d'excellentes observations de Jacqueline de Romilly dans Romilly-Vernant, 2000, p. 24.

22 Debidour, 1962, p. 81.

23 Rey, 2007, p. 167.

24 Dans ses Tusculanes (II, XI, 26), Cicéron oppose deux philosophes qu'il a connus en Grèce : l'un récitait les vers comme s'il dictait un texte, l'autre faisait sentir la quantité (numerum). D'autre part dans De l'arrangement des mots (VI, 18, 1), Denys d'Halicarnasse confesse n'aborder " des problèmes de rythmique et de métrique que contraint et forcé ». Ces deux passages montrent combien il était difficile à l'époque romaine de bien sentir la poésie classique. 
Delphes n'était vraiment très ancien. L'omphalos est donc cette pierre arrondie qui symbolise le nombril du monde. Ceux qui ont été découverts sont respectivement en calcaire gris et en marbre, et sont considérés comme des copies. J'ignorais que l'omphalos authentique avait été découvert lui aussi, en 1913. Il s'agit d'une pierre de poros, grossièrement taillée, de forme conique, haute de trente centimètres environ. Cette trouvaille, qui a fait sensation au début du siècle, ne m'aurait pas ému outre mesure si l'on ne voyait, gravé sur la pierre, orienté vers le bas, un epsilon. C'est un E maladroit, qui m'a paru infiniment plus beau que celui reproduit sur les monnaies de l'époque romaine. Je l'ai trouvé également plus vivant, peutêtre parce que, penché comme il est, il a l'air d'un animal à trois pattes. A côté de l'epsilon, on voit les lettres gamma et alpha, qui forment le nom de la déesse Terre, Ga, qui fut la toute première maîtresse du sanctuaire » (p. 251).

Ultérieurement, au chapitre 8 , dans la dernière partie du roman, le narrateur visite le musée de Delphes et pense y voir cet omphalos archaïque. Mais il ne trouve que celui en marbre d'époque romaine. Le gardien, interrogé, lui indique que l'omphalos qu'il cherche se trouve relégué dans la réserve. Survient alors le troisième savant qui, après le professeur grec Caradzoglou et le bibliothécaire de l'École française François Bouchard, vient au secours de notre amateur en antiquités delphiques. Il s'agit d'un épigraphiste français nommé Préaud qui lui fait le récit suivant : “Il m'a raconté que, dès son arrivée à Delphes, il s'était intéressé à l'omphalos archaïque... Il avait d'abord été intrigué par le couteau, qui était resté coincé dans un trou traversant la pierre de haut en bas, et que ses prédécesseurs n'avaient jugé utile de retirer. Il avait été également surpris par les traces de stuc que portait l'omphalos. Il l'a donc lavé copieusement, ce qui a fait apparaître un peu mieux l'inscription : il a constaté que la longue ligne horizontale soutenue par trois petites verticales, l'E couché, se composait en fait de deux pi majuscules $(\Pi)$ entre lesquels se cachait un alpha. Il a lu la syllabe “ $P A P$ ». Il a reconnu un autre alpha un peu plus à droite. Le gamma $(\Gamma)$ en déséquilibre était un lambda $(\Lambda)$, et l'alpha du mot $\Gamma$ A, la Terre, un omicron $(\mathrm{O})$ et un upsilon $(\mathrm{Y})$ ligaturés, un " $O U$ ». Il a lu, en fin de compte, «PAPALOU». Comme une famille du nom de Papaloukas a existé à Delphes, il n'était pas difficile de deviner qu'un de ses membres avait gravé son nom sur la pierre. Le couteau non plus n'était pas très ancien : sur la lame était gravée la date 1860. Il pense que la pierre couronnait à l'origine une de ces pieuses constructions, dédiées à un saint, semblables aux calvaires qui poussent au bord des routes grecques, et qu'une croix de fer était scellée dans le trou » (p. 333-334). Or un lecteur curieux, helléniste de surcroît, qui, effectue, parallèlement au narrateur, quelques recherches sur l'omphalos delphique, s'avise assez vite que le récit de l'épigraphiste fictif reprend en fait les résultats d'une étude publiée en 1951 par un archéologue français ${ }^{25}$. À partir 
des années 1950, dans les milieux de spécialistes, l'affaire est entendue, le sort de l'«omphalos archaïque » est scellé26.

Les réflexions que le récit de l'épigraphiste suggère au narrateur nous éloignent de l'univers intellectuel des archéologues et renouent avec la pensée et le style de l'auteur qui réfléchit dans son roman de façon autonome, personnelle et critique sur la place de Delphes dans la littérature néo-hellénique : “ Son récit m’a révélé un aspect de l'E auquel je n'avais pas songé, son caractère ironique. « L'epsilon se moque de ses adeptes, ai-je pensé. Il se plaît à induire en erreur même les plus érudits d'entre eux ». Cet E trompeur, exprimait peut-être mieux la Pythie et son dieu que la lettre mystique gravement commentée par Plutarque. Je me suis souvenu de l'ambiguïté des oracles. On dit que la Pythie a conseillé à Néron de redouter la soixante-treizième année - Cavafy mentionne cet avertissement dans un de ses poèmes. Néron s'est senti soulagé car il était encore jeune - cependant l'homme qui allait le renverser avait déjà soixante-treize $a^{27}{ }^{27} \ldots$ J'ai pensé aussi à Sikélianos, qui n'ignorait sans doute pas l'existence de cet omphalos et qui devait le croire authentique ${ }^{28}$. La métamorphose du vénérable objet en vulgaire pierre néohellénique semblait railler non seulement le romantisme des archéologues, mais aussi la nostalgie des Grecs pour leur passé » (p. 334).

\section{Épilogue}

Nous avons vu dans la première section de cette étude que le narrateur de La langue maternelle, tout en confessant que le grec de l'Iliade lui était incompréhensible, restait sensible au fait que beaucoup de mots n'avaient guère changé au cours de la longue histoire de la langue grecque. De façon analogue, le

26 Mais il figure encore par exemple dans l'édition du Guide Bleu en 1967 (p. 641). L'éditeur du traité de Plutarque dans la Loeb Classical Library pouvait écrire dans la première édition (1936) : " This character (scil., the epsilon) has been found on the old omphalos discovered in 1913 at Delphi in the temple of Apollo. » (vol. V, p. 196), mais cela n'a pas été corrigé dans les réimpressions ultérieures jusqu'en 1984, date de l'édition que j'ai consultée.

27 Il s'agit du poème intitulé Délai accordé à Néron qui se termine ainsi : “ Mais, en Espagne, Galba rassemble et exerce en secret son armée - lui, le vieillard de soixantetreize ans » (trad. Marguerite Yourcenar).

28 Le poète Anghélos Sikélianos (1884-1951), à supposer qu'il se soit intéressé à l'omphalos archaïque, ne vécut pas assez longtemps pour être le témoin éventuel de son déclassement en simple pierre de Papaloukas. Plus haut dans le roman (p. 182), le narrateur avait déjà dit tout le mal qu'il pense de son ton " prophétique, pompeux » et de son “ idée delphique », concrétisée en particulier par des fêtes organisées à Delphes en 1927 et 1930. 
poète Georges Séféris, couronné par le prix Nobel de littérature en 1963, dans une conférence prononcée à Stockholm à l'occasion de la remise de ce prix, pouvait

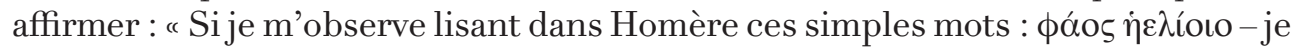
dis aujourd'hui $\phi \hat{s}$ tov̂ s'apparente plutôt à une psyché collective qu'à un effort du savoir. C'est une note, pour ainsi dire, dont les harmoniques s'étendent bien loin, cela est d'un toucher bien différent de ce que peut donner une traduction. Car enfin nous parlons la même langue - et le sentiment d'une langue relève aussi bien de l'émotion que du savoir. Une langue altérée, si vous voulez, par une évolution plusieurs fois millénaire, mais malgré tout fidèle à elle-même »29.

Cette communauté de vue relative à la langue grecque est d'autant plus remarquable que le ton des deux auteurs est dans l'ensemble fort différent. Alors que Séféris dans ses Essais s'exprime en grand lettré, ne s'autorisant à l'occasion que quelques remarques critiques sur les excès du culte de l'antiquité ${ }^{30}$, Vassilis Alexakis donne un tour beaucoup plus ouvertement polémique à sa critique de l'anticomanie de ses compatriotes, critique faite dans l'optique du " grec moyen » plus proche de la culture populaire que de la culture savante. Dans un entretien déjà cité dans le prologue, l'auteur déclare : «La langue maternelle, qui est un livre si important pour moi, n'a pas eu le succès qu'il aurait dû avoir, à mon avis, en Grèce. Peut-être parce que je me moque un peu de l'Antiquité, et de l'éducation grecque ». Mais il dit aussi un peu plus loin : “ Au cœur de La langue maternelle, il y a la langue grecque et la Grèce classique „31. En fait beaucoup de lettrés grecs, tout en sachant prendre une juste distance par rapport à un culte de l'antiquité qui est une sorte d'idéologie nationale, jettent sur la Grèce antique un regard différent de celui que peut avoir un Latin, pour les raisons qu'exprime fort bien Georges Séféris dans le passage un peu plus haut cité.

Cette relation spéciale de la Grèce moderne à la Grèce ancienne peut enfin expliquer l'incompréhension de beaucoup de Grecs à l'égard des hellénistes occidentaux - Francs et Latins comme on disait naguère - qui établissent une coupure absolue entre présent et passé, limitant leurs intérêts à l'Antiquité. Dans les dernières pages de son roman, Vassilis Alexakis campe ainsi la figure d'une antiquisante tout à l'opposé du philhellène bibliothécaire de l'Ecole française :

“Une archéologue française d'une trentaine d'années est assise à ma droite.

29 SefEris, 1987, p. 86.

30 Voir par exemple Seferis, 1987, p. 140 et mes remarques dans BaLlabriga, 2009, p. 401 414 (p. 409).

31 Bessy, 2011, p. 245 et 253. Et pour sa part Marianne Bessy fait justement observer que le narrateur passe de l'ignorance à l'érudition et que l'auteur illustre ainsi « son propre désir de démontrer une connaissance personnelle approfondie de l'histoire grecque. » (Bessy, 2011, p. 224) 
Elle habite depuis quatre ans en Grèce, mais elle n’a pas appris le grec moderne. Elle en est fière » (p. 359).

Le narrateur en vient tout de même à lui conseiller quelques lectures pour l'initier à la culture grecque moderne :

« Mais dites-moi alors ce que je dois lire ! dit-elle avec empressement.

Par où commencer? Je lui cite Cavafy, il existe cinq ou six traductions de ses poèmes en français. Elle a sorti de son sac un superbe stylo, avec une plume en or.

Comment ça s'écrit?

Elle ne connaît même pas Cavafy. Elle écrit le nom du poète sur une serviette en papier qui boit l'encre et se déchire. Elle va finir par me mettre en colère, cette fille » (p. 361).

\author{
Alain BaLLABRIGA \\ Directeur de recherche honoraire \\ (CNRS) \\ Le Clos Fleuri \\ 57, chemin de la Pélude \\ 31400 Toulouse \\ a.ballabriga@gmail.com
}

\title{
Ouvrages cités
}

Alexakis, V., 1979 : Les Grecs d'aujourd'hui, Paris.

Ballabriga, A., 2006 : “Philhellénisme et prononciation du grec », Anabases 3, p. 57-77.

Ballabriga, A., 2009 : « Séféris et Homère », Pallas, 79, p. 401-414.

Bessy, M., 2011: Vassilis Alexakis. Exorciser l'exil, Amsterdam/New York.

Bousquet, J., 1951 : “ Observations sur l' 'omphalos archaïque' de Delphes », BCH, 75, p. 210-223.

Debidour, V.-H., 1962 : Aristophane par lui-même, Paris.

Koukoules, M., 1983: Loose-Tongued Greeks. A Miscellany of Neo-Hellenic Erotic Folklore, Paris.

Rey, A., 2007 : L'amour du français. Contre les puristes et autres censeurs de la langue, Paris.

Romilly de, J., Vernant, J.-P., 2000 : Pour l'amour du grec, Paris.

Seferis, G., 1987 : Essais. Hellénisme et création. Traduction du grec, choix et présentation par Denis Kohler, Paris.

Van Steen, G., 2000: Venom in Verse. Aristophanes in Modern Greece, Princeton. 